\title{
LA CONTEXTUALIZACIÓN EN LA CIENCIA POLÍTICA: UNA PERSPECTIVA LATINOAMERICANA $^{1}$
}

\author{
M. Victoria Murillo
}

LA CONTEXTUALIZACión ES CRUCiAL para establecer teorías más amplias, pues nos obliga a especificar las condiciones del enfoque de su aplicabilidad. Incluso cuando las teorías generales son más una aspiración que una realidad en las ciencias sociales desde un punto de vista positivista, la aplicación de regularidades empíricas restringidas implica un sentido claro de "bajo qué condiciones" se consideran dichas regularidades para probar su validez externa. ${ }^{2}$ En consecuencia, la contextualización es crucial para mejorar nuestra comprensión de procesos políticos diversos y peculiares.

Sostengo aquí que el desafío primordial para la ciencia política para proveer el instrumental que ayude a entender nuestras realidades políticas actuales involucra su capacidad para lidiar con la tensión de producir teorías generales, por un lado, y la capacidad, por el otro lado, para enfocar conocimiento en contexto. Esta tensión es crucial porque requerimos de conocimiento contextualizado para ampliar nuestro aparato conceptual en un mundo asaz diverso que se enmascara tras la uniformidad aparente de la "globalización" o inclusive de los fenómenos "globales". Paradójicamente,

${ }^{1}$ Agradezco los comentarios de Karen Barkey, Juan Pablo Luna, Andrew Schrank, Juan Olmeda y de un dictaminador anónimo, así como a los participantes en el seminario en El Colegio de México.

${ }^{2}$ Reconozco que otras tradiciones epistemológicas podrían tener preocupaciones diferentes y estén formadas originalmente en la tradición del "ensayismo". Con todo, escribo este trabajo desde una perspectiva positivista que otros colegas en la disciplina podrán compartir o no. 
mientras que la "globalización" incrementa la urgencia de resolver esta tensión, la difusión "global" de la ciencia política estadounidense suscita incentivos que vuelven más difícil esta labor. ${ }^{3}$

\section{GLOBALIZACIÓN Y CONOCIMIENTO CONTEXTUAL EN CIENCIA POLÍTICA}

Pese al interés de la ciencia política, y en especial de la economía política internacional, para entender la "globalización" económica, se ha dado poca atención a la manera como este proceso afecta la disciplina. ${ }^{4}$ La velocidad e interconexión del cambio producido por lo que llamamos "globalización" aumenta la urgencia de resolver la tensión entre generalización y la contextualización. (Con todo, reconozco que la ciencia política ha tenido dificultades para explicar los fenómenos "globales" más añejos. ${ }^{5}$ ) Aún así, la difusión global de los incentivos disciplinarios generados por la ciencia política en Estados Unidos puede limitar nuestra capacidad de atender esta tensión. Estos incentivos llevaron a la ciencia política estadounidense en una dirección que descuida el conocimiento

${ }^{3}$ Mi caso sobre la importancia de la "contextualización" está en buena medida motivado por mi trabajo como comparativista y por mi investigación sobre América Latina, así como por mi trayectoria personal desde que me convertí en científica social en la Universidad de Buenos Aires, si bien recibí mi grado doctoral en Estados Unidos. Enseño principalmente en Estados Unidos e investigo sobre América Latina. Más aún, mi interés en esta región motiva mi propio impulso normativo hacia la ciencia política, conque el enfoque en este trabajo atañe a la política comparada en la política latinoamericana.

${ }^{4}$ El término "globalización" remite por lo común a Theodore Levitt, quien lo popularizó en su artículo "Globalization of Markets", aunque el término se había empleado antes. Véase Theodore Levitt, "Globalization of Markets”, Harvard Business Review, mayo-junio de 1983.

${ }^{5}$ Por ejemplo, la ciencia política no tiene una teoría aceptada para explicar la efervescencia mundial de la década de 1960 y sus distintas manifestaciones, desde el Flower Power a la guerra de guerrillas y los movimientos reformistas pacíficos. Tampoco provee de una sabiduría convencional para rendir cuentas por la esclavitud y la diversidad de sus manifestaciones y evolución alrededor del mundo. 
contextualizado, a la vez que su difusión global puede extender esta limitación.

La evolución de la ciencia política estadounidense en la era de la posguerra se caracterizó por una atención exacerbada hacia el resto del mundo con la aparición de la política comparada y los estudios de área. En particular, las aspiraciones políticas estadounidenses se reflejaron en la academia con la "teoría de la modernización" como un esfuerzo normativo para conducir la democracia capitalista a lo que se etiquetó como el mundo "menos desarrollado". La base "científica" de este esfuerzo se consolidó durante la revolución behaviorista, que desplazó al institucionalismo formal en ciencia política. ${ }^{6}$ Las conexiones con la sociología y la economía del desarrollo produjeron sinergias en programas interdisciplinarios enfocados en los estudios internacionales o de área, que financiaba Estados Unidos por medio de un gobierno ávido de información para alimentar su política exterior durante la Guerra Fría. Los estudios de área destacaron la inmersión en el caso incluso cuando se empleaba el lente unidireccional de la "modernización" para enfocarse en contextos cuyos elementos ofrecieron ya apoyo para el statu quo "tradicional", ya para el esfuerzo de "modernización", con poca atención a su conexión a las relaciones de poder internacionales. Se suponía que el punto de llegada de estas trayectorias sería el mismo, incluso si Estados Unidos y Europa habían llegado antes e incluso si cada experiencia no suponía estar conectada con las otras.

Las críticas de la teoría de la "modernización" se habían difundido en América Latina, no sorpresivamente, pues la Guerra Fría había impuesto la hegemonía estadounidense en esta región. La "modernización" defendía una lectura particular de la política interna de sus países y su trayectoria esperada hacia el capitalismo y la democracia, especialmente luego de que la Revolución cubana había parecido alterar este orden y de que la "teoría de la modernización” se asociara a las políticas promovidas por la Alianza para el

${ }^{6}$ Un libro fundamental para el planteamiento de esta agenda empírica para la teoría de la modernización es Gabriel Almond y Sidney Verba, Civic Culture, Londres, Sage, 1963. 
Progreso en la región. En América Latina, las ideas económicas marxistas y estructuralistas sostenían críticas a la "modernización", lo que era crucial en la generación de la "teoría de la dependencia".7 La teoría de la dependencia enfatizaba la interconexión involucrada en las relaciones de poder entre países y destacaba la posibilidad del "subdesarrollo" a la vez que distinguía los diferentes tipos de "periferia" que habían surgido en América Latina dada la relación entre élites internas y mercados internacionales. La teoría de la dependencia fue muy influyente en la academia latinoamericana de la década de 1970 , pero no lo fue tanto en la ciencia política estadounidense. ${ }^{8}$ Otra revolución intelectual -ligada también a ideas económicas- sucedía en la ciencia política estadounidense en los setenta, mientras los latinoamericanistas discutían la teoría de la dependencia. Las instituciones reactivaron la ciencia política en la forma de la elección racional y del institucionalismo histórico. Ambas ramas del "nuevo institucionalismo" en la ciencia política se enfocaban en la manera como las instituciones constriñen la acción humana.

El enfoque sobre el contexto institucional se realizó a expensas de la cultura a la vez que facilitaba las teorías generalizables sobre el impacto de las instituciones en países y contextos variados. Las teorías generales comenzaron desplazando el conocimiento contextual conforme la nueva teoría institucionalista tendía a asumir que las instituciones formales similares operaban de la misma forma en contextos diversos. Este nuevo aparato teórico incrementó nuestro conocimiento sobre las acciones estratégicas, el cual destacaba la voluntad de los actores dentro de los

${ }^{7}$ Fernando Henrique Cardoso y Enzo Falleto, Dependencia y desarrollo en América Latina, México, Siglo XXI Editores, 1969.

${ }^{8}$ La obra relacionada de Immanuel Wallerstein (The Modern World System, Academic Press, 1974) fue más influyente en relaciones internacionales que en política comparada. Por su parte, los trabajos de Peter Evans (Dependent Development: The Alliance of Multinational, State and Local Capital in Brazil, Princeton, Princeton University Press, 1979) y de Guillermo O'Donnell (Modernization and Bureaucratic Authoritarianism: Studies on South American Politics, Berkeley, University of California Press, 1973) sobre la teoría de la dependencia tuvieron gran impacto en el estudio de América Latina y en la política comparada en general. 
límites impuestos por las instituciones. Con todo, no proveyó de instrumental para comprender el origen y la evolución de las preferencias políticas que habrían de ser contenidas por las instituciones. ${ }^{9}$

Este nuevo giro científico que aportaba el institucionalismo de la elección racional (su variante histórica se ocupaba más de la evolución histórica) se asociaba también a la política exterior estadounidense. Ya en la década de 1990, las políticas e instituciones promovieron el "Consenso de Washington" que se prometió para generar incentivos que sancionaran la acción humana conque se facilitase el "desarrollo económico". La condicionalidad económica y política se asociaba a los esfuerzos de ingeniería humana que permitirían que América Latina (y otros países "en desarrollo") tuvieran mercados prósperos y democracia, si bien este último efecto era más relevante fuera de la región, ya que América Latina había experimentado un proceso endógeno de democratización, como se discute más abajo. En la aplicación del nuevo institucionalismo al mundo de las políticas, decayó la atención al contexto local, especialmente en tanto que los economistas dominaban la asesoría sobre política exterior. Como resultado, las consecuencias de las reformas orientadas hacia el mercado no fueron lo que (formalmente) se esperaba, especialmente en las regiones, como América Latina, donde la debilidad institucional permitía el cumplimiento dispar e intercambio en serie de instituciones adoptadas. ${ }^{10}$

Simultáneamente, la economía política comparada buscaba entender la interconexión global que iba en aumento a causa de la movilidad de capitales, el comercio creciente, el rápido cambio

9 Aludo aquí a Peter Hall, "The Dilemmas of Contemporary Social Science", Boundary 2, vol. 34, núm. 3, otoño de 2007. Esta falta de conocimiento de las preferencias políticas no implica un regreso a los estudios culturales como la única solución, aunque sea un camino que habrá de tomarse, sino un énfasis más general en la exploración de sus variaciones entre las culturas, contextos y otras características humanas que pueden mejorar nuestro conocimiento del comportamiento político, en vez de asumir sin más dichas preferencias.

${ }^{10}$ Steven Levitsky y M. Victoria Murillo, "The Causes and Implications of Variation in Institutional Strength”, Annual Review of Political Science, vol. 12, 2009. 
tecnológico en la velocidad y calidad de las comunicaciones. La generalización predominaba también en modelos sofisticados de difusión y en análisis diádicos sobre las conexiones entre pares de Estados. Incluso un fenómeno contextual decisivo, como la aparición de la Unión Europea, se interpretaba a veces como parte de una tendencia global que anunciaba la desaparición del Estado nación. Para ser justos, como reacción a la idea de una convergencia global, las economías políticas regionales aún se analizaban, tanto en el contexto de los países industrializados como en respuesta de América Latina a los retos que trajo el "Consenso de Washington". ${ }^{11}$

Conforme el nuevo institucionalismo reemplazó la teoría de la modernización, la promesa de la democratización comenzó a cumplirse. La tercera ola de la democratización se movió fuera de los avanzados países industrializados, mientras que la Guerra Fría terminaba con el colapso del Comunismo. La ciencia política falló en pronosticar o proveer de explicaciones ex post aceptables para estos dramáticos eventos, que parecían confirmar la teoría de la "modernización": prevalecieron el desarrollo capitalista y la democracia. Fukuyama sugirió incluso un posible "fin de la historia". ${ }^{12}$ Los instrumentos que el nuevo institucionalismo aportaba eran insuficientes para producir sabiduría convencional para la democratización, aun cuando ofrecían marcos útiles para la comprensión de las instituciones democráticas funcionales.

La difundida naturaleza de la integración económica, el cambio tecnológico, la expansión del desarrollo capitalista y -en menor medida- las elecciones democráticas nos abrieron el paso al

${ }^{11}$ Peter Hall y David Soskice (Varieties of Capitalism, Oxford, Oxford University Press, 2001) abren toda una bibliografía sobre la importancia de contextualizar la globalización en países industriales avanzados. Entre los latinoamericanistas había una vasta bibliografía de economía política sobre reformas de políticas de mercado y sus consecuencias, tanto en el nivel nacional como en el subnacional. Un estudio comparativo decisivo de las reformas del mercado en la región, financiado por la CEPAL, se compendia en Barbara Stallings y Wilson Peres, Crecimiento, empleo y equidad: el impacto de las reformas economicas en America Latina y el Caribe, México, FCE / CEPAL, 2000.

${ }^{12}$ Francis Fukuyama, “The End of History?”, The National Interest, verano de 1989. 
nuevo milenio marcado por el gran abrazo de la "globalización". Inclusive los conflictos que dejaron de estar conectados a la Guerra Fría (por lo común caracterizados de sectarios o étnicos en otra conceptualización general más) podían describirse como reacciones ludistas a la globalización o consecuencias de la debilidad de los Estados nación. Como fuera, el ataque a las Torres Gemelas cambió el enfoque de la política exterior de Estados Unidos y la atención de la academia estadounidense a lo que Huntington ya había llamado el "choque de civilizaciones". ${ }^{13}$

El impacto interno del terrorismo y el impacto externo del conflicto internacional que no podía definirse por los Estados nación señaló la entrada al nuevo milenio para la ciencia política, lo que renovó el interés en el conflicto, la etnicidad y la religión, mientras que al mismo tiempo buscaba apoyo "científico" (y financiero) para sus investigaciones. Como antes, la ciencia política buscaba inspiración en la economía, especialmente en la economía y neuroeconomía conductuales. Mientras que el enfoque sobre la identificación causal presentaba incentivos para la innovación metodológica por efecto de variables instrumentales y regresión discontinua, el impulso más fuerte en esa dirección se recibía del giro hacia los experimentos de campo. ${ }^{14}$ De hecho, los creyentes más firmes de esta nueva metodología llegaron a desconfiar de la posibilidad de aprender de los datos "observacionales". ${ }^{15}$ Más aún, el experimentalismo prometía una metodología para generar políticas basada en la evidencia e intervenciones exitosas para modificar el mundo. Mientras que se trabajaba en algunas de esas promesas, esta nueva tendencia suscitó también incentivos que reforzaban el descuido por la contextualización y restringían las cuestiones consideradas por los estudiantes de posgrado dados los límites éticos y prácticos de la experimentación en ciencias sociales.

13 Samuel Huntington, "The Clash of Civilizations?", Foreign Affairs, vol. 72, núm. 3, verano de 1993.

${ }^{14}$ Macartan Humphreys y Jeremy Weinstein, "Field Experiments and the Political Economy of Development”, Annual Review of Political Science, vol. 12, 2009.

${ }^{15}$ Alan Gerber, Donald Green y Edward Kaplan, "The Illusion of Learning from Observational Research", en Ian Shapiro, Rogers Smith y Tarek Masoud (eds.), Problems and Methods in the Study of Politics, Cambridge, Cambridge University Press, 2014. 
El énfasis en el método es particularmente preocupante en este caso dada la limitación para llevar a cabo experimentos de campo para el estudio de muchos asuntos importantes en ciencia política, como las guerras, revoluciones, recesiones, democratización y golpes militares, por nombrar sólo algunos. ${ }^{16}$ En efecto, las preguntas sin respuesta de las causas de la Guerra Fría o de la Tercera Ola de Democratización no parecen acomodarse a esta metodología. Adicionalmente, las afirmaciones sobre los hallazgos experimentales de la ciencia política no siempre atienden apropiadamente la validez externa, aunque la variación producida por el contexto pueda ser mayor que aquella generada por fuentes de heterogeneidad ya identificadas, incluso en temas bien estudiados. ${ }^{17}$ Esta preocupación podría aumentar con la concentración de experimentos en países concretos, dados los costos y las implicaciones prácticas. ${ }^{18}$ Finalmente, con estas limitaciones, la promesa de la ciencia política, de políticas basadas en evidencias, se arriesga a presentar solamente intervenciones localizadas, que no dan herramientas a los diseñadores de políticas para lidiar con los problemas más apremiantes en sus países ni consideran que los seres humanos reaccionan estratégicamente a las intervenciones reduciendo su impacto una vez que la población afectada las conoce.

Un fenómeno paralelo ha aumentado estos procesos en la disciplina. La evolución del sistema universitario estadounidense ha intensificado los incentivos que operan en contra de la contextualización en la ciencia política. Los estímulos financieros llevaron a los programas de posgrado a reducir el tiempo de terminación, lo que junto a la necesidad de mayor formación requerida en los nuevos métodos se opone a dedicar tiempo en el campo de trabajo. Hoy en día, los estudiantes de posgrado y los profesores jóvenes

${ }^{16}$ Ian Shapiro, "Los métodos son como las personas: si te enfocas sólo en lo que no pueden hacer, te decepcionarás”, en Shapiro, Smith y Masoud (eds.), op. cit.

${ }^{17}$ Lant Princhett y Justin Sandefur, "Context Matters for Size: Why External Validity Claims and Development Practice Don't Mix”, Center for Global Development, Washington, D. C. Reportan además resultados diferentes incluso para la misma intervención en el mismo país cuando se involucran organizaciones diversas.

${ }^{18}$ Graeme Blair, Radha Iyengar y Jacob Shapiro, "Sample Bias in the Experimental Movement in Economics and Politicals Science", documento de trabajo. 
que buscan crecer profesionalmente tienen fuertes alicientes para confiar en los conjuntos de datos existentes y en la Internet, en vez de hacer estudios de campo para recoger datos cuantitativos y cualitativos, lo que requiere formación de conocimientos y tiempo para investigarlo en el campo. De esta manera, hay un costo a pagar para los académicos que buscan invertir en el conocimiento y en las herramientas necesarias para la "contextualización", lo que no se reconoce necesariamente en los medios de publicación, ni por los colegas, ni aun por los decanos que buscan abreviar el tiempo de término.

En breve, la ciencia política, como disciplina en Estados Unidos, ha avanzado en términos de la exploración de métodos nuevos, lo que abrió las puertas a nuestra comprensión de un mundo cambiante. Con todo, no está claro si la innovación metodológica se ha aplicado siempre de un modo que pueda conducirnos a lo que Peter Hall señala como el cometido crucial de la disciplina: "no debemos emprender generalizaciones ni escribir sólo historias locales. Pero la generalización válida sobre tales asuntos demanda atención a los imaginarios políticos de tiempos y lugares precisos, y a los procesos que los institucionalizan con el tiempo. Los modelos desarrollados por analistas cuya familiaridad con los países que estudian se basa en un sobrevuelo difícilmente bastarán". ${ }^{19}$ Es en este tenor que me vuelvo ahora a la globalización de la ciencia política y a su despliegue en América Latina.

\section{La globalización de la Ciencia POLÍ́tica en América Latina}

Mientras que la velocidad de la interconexión del cambio aumenta con la globalización, la desatención a la contextualización en ciencia política también se propagó como un resultado involuntario de la globalización. Desde 1995, la educación se había clasificado como un servicio protegido bajo el Acuerdo General de Servicios de la Organización Mundial de Comercio. Este acuerdo redujo las barreras comerciales al simplificar la "oferta de cursos en línea en países

${ }^{19}$ Peter Hall, “The Dilemmas of Contemporary Social Science”, p. 16. 
extranjeros, facilitar los campus filiales y satelitales, y hacer posible el movimiento de servicios de estudiantes, profesores e investigadores". ${ }^{20}$ Por consiguiente, el modelo de universidad estadounidense se ha extendido rápidamente en partes de Asia y especialmente en el Medio Oriente, conforme abrieron campus estadounidenses y se fundaron universidades diseñadas según las escuelas de Estados Unidos. ${ }^{21}$ Fuerzas más sutiles entraron en juego en América Latina, donde los sistemas universitarios se fundaron de acuerdo al modelo europeo y donde la búsqueda de ideas originadas en Estados Unidos y Europa era generalizada desde las independencias.

América Latina existe como una región debida a un fenómeno global anterior, la expansión de Europa Occidental en el resto del mundo o la conquista de las poblaciones nativas americanas por parte de los europeos desde 1492. La parte ocupada por España tuvo muchas conexiones a causa de la imposición del gobierno unificado, la religión y el lenguaje. Pese al mercantilismo español, las élites ya nativas (que no tenían los mismos privilegios que sus pares metropolitanos) buscaron ideas de los países de Europa Occidental y de Estados Unidos. La invasión napoleónica en España facilitó la independencia de las colonias, guiada por esas mismas élites hacia nuevas repúblicas a comienzos de la descolonización. Estados Unidos figuraba de manera prominente como modelo para estas élites, de modo que se adoptaron constituciones presidencialistas a lo largo de la región. Al lidiar con poblaciones de mayoría no blanca y altos niveles de inequidad que amenazaban sus privilegios, estas élites confiaban en un cumplimiento dispar de los derechos constitucionales a la vez que eran propensos a los conflictos que acababan en cambios múltiples del orden. ${ }^{22}$

${ }^{20}$ Isaac Kamola y Neema Noori, "Higher Education and World Politics", PS, vol. 47, núm. 3, julio de 2014, p. 599.

${ }^{21}$ Neema Noori, "Does Academic Freedom Globalize? The Diffusion of the American Model of Educacion to the Middle East and Academic Freedom", PS, vol. 47, núm. 3, julio de 2014.

${ }^{22}$ Steven Levitsky y M. Victoria Murillo, "Building Institutions on Weak Foundations: Lessons from Latin America”, en Daniel Brinks, Marcelo Leiras y Scott Mainwaring (eds.), Reflections on Uneven Democracies: The Legacy of Guillermo O'Donnell, Baltimore, The Johns Hopkins University Press, 2014. 
Luego del fin de las guerras civiles que siguieron a las independencias, América Latina se unió a la economía "global". Producía materias primas para los países industrializados, recibía inversión extranjera directa para generar la infraestructura necesaria para sostener este comercio y, en el Cono Sur, atraía una afluencia de migrantes europeos que se esperaba "blanquearan" a las poblaciones locales. América Latina había sido tomada de lleno en la primera ola de desarrollo capitalista. Mientras tanto, el "patio trasero" de Estados Unidos en América Central y el Caribe iba a determinarse de manera dramática por el colonialismo directo y la intervención del nuevo imperio estadounidense antes del nacimiento de la teoría de la "modernización". La ubicación de América Latina en el área de influencia de Estados Unidos durante la Guerra Fría estaba íntimamente asociada a la desconfianza ante la teoría de la "modernización" y la aparición de ideas económicas y políticas alternativas en las ciencias sociales latinoamericanas, como se mencionó antes.

La Globalización, ahora oficialmente con G mayúscula, llegó de nuevo a la región, tras los pasos de dos procesos endógenos que perfilaron su impacto. Políticamente, América Latina se incorporó a la Tercera Ola de democratización a finales de la década de 1970, tomó fuerza en los años ochenta y culminó en los noventa. Económicamente, la escasez de capital producida por la crisis de deuda de 1982 incrementó su exposición a las presiones del "Consenso de Washington" para las reformas extendidas orientadas hacia el mercado.

El impacto de la democratización no sólo afectó las posibilidades de millones de ciudadanos cuyos derechos iban a expandirse (si bien desigualmente), sino que remodeló además dramáticamente las ciencias sociales en la región. La ciencia política redescubrió la contingencia de la acción humana y su rol determinante en el éxito de las transiciones democráticas ante las condiciones estructurales inhóspitas de la región. ${ }^{23} \mathrm{El}$ redescubrimiento de la

${ }^{23}$ Conviene traer a colación dos foros cruciales que explican este cambio intelectual. Primero, el Social Science Research Council y su proyecto sobre transiciones a la democracia a inicios de los ochenta ofreció un espacio fundamental 
"autonomía de la política" en la ciencia política latinoamericana fue crucial para entender la democratización en una región con altos niveles de inequidad en el ingreso aun antes de la Caída del Muro de Berlín. ${ }^{24}$

Pero la Tercera Ola de democratización no sólo apuntaló la fe de los académicos en la autonomía de la política. Trajo de vuelta a América Latina a la corriente principal de la ciencia política en general, pues América Latina se jacta de más de una docena de democracias presidenciales, más que cualquier otra región del mundo, y su consolidación abrió la puerta al estudio de las instituciones, las elecciones y los partidos políticos en ambientes nuevos y distintos. El predominio del presidencialismo, en particular, permitió a los especialistas importar teorías de Estados Unidos sin prestar siempre atención a los factores contextuales. ${ }^{25}$

La tendencia a reducir el contexto a elementos institucionales y a aplicar teorías generales sin contextualización se agudizó entonces junto a la esperanza en la acción estratégica que atraería la consolidación de las democracias nuevas y débiles. Es importante notar, sin embargo, dos eventos prometedores hacia la contextualización

para los académicos exiliados de la región para estudiar el proceso de la dictadura y su evolución potencial hacia las transiciones a la democracia, como lo testifican los volúmenes que produjo (Transitions from Authoritarian Rule, Baltimore, The Johns Hopkins University Press, 1986). En segundo lugar, las instituciones mexicanas de alto rendimiento, como El Colegio de México, el cide, Flacso y la unam, también recibieron a especialistas latinoamericanos exiliados, con que abrieron un nuevo espacio determinante de debate intelectual sobre la dictadura y la democracia en la región. En esos debates interrelacionados, el costo de las experiencias anteriores de cambio radical y la represión subsecuente de las dictaduras militares, con la persecución y el exilio de muchos intelectuales latinoamericanos, fue decisivo para resaltar la importancia de la democracia como garante de los derechos humanos y el orden de la ley.

${ }^{24}$ Guillermo O'Donnell y Phillip Schmitter, Transitions from Authoritarian Rule: Tentative Conclusions about Uncertain Democracies, Baltimore, Johns Hopkins University Press, 1986.

${ }^{25}$ Juan Pablo Luna, María Victoria Murillo y Andrew Schrank, "Latin American Political Economy: Making Sense of a New Reality", Latin American Politics and Society, vol. 56, núm. 1, p. 30. 
en el estudio de las democracias latinoamericanas en la academia estadounidense: los estudios sobre la variación subnacional institucional y su dinámica, especialmente en grandes países federados, y los estudios que buscan entender la diversidad de las prácticas electorales entre votantes heterogéneos y en distintos partidos políticos. ${ }^{26}$

Simultáneo a la democratización, sobrevino en la región un impacto que limitó la acción humana: la crisis de deuda. El padecimiento que en la población de países recién democratizados infligió el ajuste económico de los ochenta y noventa hace aún más notable el éxito del proyecto endógeno. La escasez de capital dio poca oportunidad a los gobiernos democráticos latinoamericanos, que en su mayoría se incorporaron a la ola orientada hacia el mercado que promovía el Consenso de Washington, si bien a distinto ritmo y con distintos patrones de implementación. Los especialistas en América Latina, ya en la región, ya en Estados Unidos, se ocuparon de aquellos procesos y su irregularidad al enfocarse en las políticas de las reformas neoliberales y en sus consecuencias, aduciendo algo de contextualización, de la misma forma que en los estudios de economía política europea ya mencionados.

${ }^{26}$ A riesgo de olvidar muchos exponentes importantes, mencionaré sólo dos ejemplos de la crítica comparada sobre patrones subnacionales de tipos distintos: Edward Gibson, Boundary Control: Subnational Authorianism in Federal Democracies, Cambridge University Press, 2012; y Tulia Falleti, Decentralization and Subnational Politics in Latin America, Cambridge University Press, 2010. Estos son solamente dos ejemplos de una bibliografía mucho más vasta. De la misma manera, es muy amplio el trabajo sobre nexos políticos, clientelismo y diversificación de la agenda de los políticos latinoamericanos. Los primeros ejemplos de esta discusión son Javier Auyero, Poor People's Politics, Durham, Duke, 2000; y Steven Levitsky, Transforming Labor-Based Parties in Latin America, Cambridge University Press, 2003; Ernesto Calvo y María Victoria Murillo, "Who Delivers? Partisan Clients in the Argentine Electoral Market?", American Journal of Political Science, vol. 48, núm. 4, 2004, pp. 742-757; y Alberto Díaz Cayeros, Beatriz Magaloni y Federico Estévez, "The Erosion of Party Hegemony, Clientelism and Portfolio Diversification: The Programa Nacional de Solidaridad (Pronasol) in Mexico”, en Herbert Kitschelt y Steve Wilkinson (eds.), Patrons or Policies? Patterns of Democratic Accountability and Political Competition, Cambridge, Cambridge University Press, 2007. 
La falta de acción que las presiones económicas externas producían recordó a América Latina sobre su honda interconexión con el resto del mundo, que en el nuevo milenio generará incentivos para un cambio dramático en la estrategia económica. ${ }^{27} \mathrm{El}$ ascenso de Asia redujo de nuevo a Sudamérica a la producción de materias primas para estos nuevos mercados y dio recursos a los gobiernos democráticos (en su mayoría de izquierda) para efectuar una redistribución a sus ciudadanos, lo que no había sido posible en los tiempos duros del ajuste económico. Centroamérica y México, en contraste, reforzaron su integración a Estados Unidos, por medio del comercio, manufacturas y remesas de migrantes. Acaso, por lo anterior, sea poco probable que observemos populismo radical en esta área. De aquí que la interconexión de América Latina con el mundo esté críticamente relacionada a los cambios temporales registrados en la región; pero también es parte de lo que contribuye a la diversidad dentro de la región. Con esto, necesitamos teorías contextualizadas que funcionen para las relaciones en general, pero que también expliquen las condiciones que resultan en experiencias diversas. La evolución de la ciencia política en la región, con todo, puede atraer incentivos que vuelven esta labor más difícil de lograr.

La combinación de democracia y crecimiento orientado por las materias primas no sólo redujo la inequidad, sino que generó una demanda creciente de educación universitaria en la región. Esta demanda dio lugar a un auge en el sector de la educación superior privada; también creó la necesidad en los gobiernos de establecer estándares de acreditación. Estas condiciones facilitaron la profesionalización de la disciplina a la que vez que daban recursos para estudiar en el extranjero, abrir programas de posgrado y, en los países más afluentes, financiar investigación en ciencias sociales y la educación de posgrado, que a su vez requirió de estándares. En este contexto, el modelo estadounidense se volvió muy influyente en la ciencia política, sobre todo cuando

${ }^{27}$ Maristella Svampa, “Consenso de las 'commodities' y lenguajes de valoracion en America Latina”, Nueva Sociedad, núm. 244, marzo-abril de 2013, pp. 30-34. 
las ciencias naturales dominaban las políticas de investigación y educación. De aquí que "la publicación en revistas de lengua inglesa, la colaboración con instituciones norteamericanas y la formación en Estados Unidos se valore con frecuencia más que publicar en la lengua local, colaborar con otros especialistas en América Latina u obtener doctorados en casa o en cualquier parte de la región". ${ }^{28}$

La exportación de ciencia política estadounidense hacia América Latina no puede separarse de las persistentes desigualdades en el financiamiento, incluso entre la abundancia alcanzada por el auge de las materias primas, y las trabas de las diferencias lingüísticas. El financiamiento, en particular, influye en las agendas de investigación por medio de los fondos y los acuerdos de colaboración. Sin embargo, existen en América Latina numerosos puntos de resistencia a la influencia de la ciencia política estadounidense, y su fortaleza varía según el contexto. Las experiencias de los países son distintas, entonces, según los recursos existentes y el desarrollo previo de la ciencia política. La fuerza de la herencia europea, diversa de por sí aunque con influencia particular de la ciencia política francesa, ofrece estilos alternativos de investigación en muchos países al igual que fuentes distintas de apoyo. Otros actores importantes son las instituciones regionales, como Clacso (Consejo Latinoamericano de Ciencias Sociales), que aporta financiamiento para establecer conexiones Sur-Sur, y Flacso, que organiza foros regionales para académicos con distintos tipos de trabajo. Como con la globalización económica, las opciones de políticas afectaron este proceso. La convergencia epistemológica avanza más rápido en Chile, México, Brasil y Argentina, los países más afluentes con sistemas de investigación más desarrollados e influencia mayor de las ciencias naturales. Con todo, aun en esos grandes países, abundan los focos de resistencia a la ciencia política estadounidense, que se sirven de marcos teóricos alternativos y de apoyo, de cepa europea en particular, que tienen sus propias limitaciones para producir conocimiento general contextualizado.

${ }^{28}$ Luna, Murillo y Schrank, op. cit., p. 31. 


\section{OBSERVACIONES FINALES}

La "globalización" incrementa la urgencia de un acercamiento interdisciplinario que mejore nuestro conocimiento de su impacto variado. Con todo, también ha lanzado alicientes hacia la disciplina de la ciencia política estadounidense con que se desluce aquella investigación para los académicos que quieren avanzar en su carrera.

A pesar de estos desafíos, deseo concluir con una nota positiva. Los debates en la disciplina sobre el tipo de ciencia política que queremos siguen ocupándose de la importancia de entender el mundo. Nuevos métodos han aportado una contribución importante para entenderlo, pese a sus limitaciones. En efecto, los métodos habían cambiado, pero el debate sobre la necesidad de una ciencia social interdisciplinaria e inductiva, guiada por los problemas más que por la teoría o el método, nunca se ha detenido. Además, la búsqueda de evidencia en la evaluación de políticas (o confrontación de políticas) es una meta loable; y la necesidad de una investigación sistemática para alcanzar dichas metas está generalizada en la ciencia política. Con todo, no puede dejar de subrayarse la importancia del contexto, si es que habremos de desarrollar conocimiento que responda por un mundo que los seres humanos intentan transformar. La contextualización puede mitigar la tendencia a la generalización de las teorías y políticas de "talla única", a la vez que aumenta el enfoque de su aplicabilidad, si es que entendemos cómo el contexto define los procesos políticos y estamos preparados para enfrentar el hecho de que los seres humanos pueden reaccionar estratégicamente al conocimiento que producimos.

Traducción de Gabriel Ramos

\section{Bibliografía}

Almond, Gabriel y Sidney Verba, Civic Culture, Londres, Sage, 1963. Auyero, Javier, Poor People's Politics, Durham, Duke, 2000. 
Blair, Graeme, Radha Iyengar y Jacob Shapiro, "Sample Bias in the Experimental Movement in Economics and Politicals Science", documento de trabajo.

Calvo, Ernesto y María Victoria Murillo, "Who Delivers? Partisan Clients in the Argentine Electoral Market?", American Journal of Political Science, vol. 48, núm. 4, 2004, pp. 742-757.

Cardoso, Fernando Henrique y Enzo Falleto, Dependencia y desarrollo en América Latina, México, Siglo XXI, 1969.

Díaz Cayeros, Alberto, Beatriz Magaloni y Federico Estévez, "The Erosion of Party Hegemony, Clientelism and Portfolio Diversification: The Programa Nacional de Solidaridad (Pronasol) in Mexico", en Herbert Kitschelt y Steve Wilkinson (eds.), Patrons or Policies? Patterns of Democratic Accountability and Political Competition, Cambridge, Cambridge, Cambridge University Press, 2007.

Evans, Peter, Dependent Development: The Alliance of Multinational, State and Local Capital in Brazil, Princeton, Princeton University Press, 1979.

Falleti, Tulia, Decentralization and Subnational Politics in Latin America, Cambridge, Cambridge University Press, 2010.

Fukuyama, Francis, “The End of History?”, The National Interest, verano de 1989.

Gerber, Alan, Donald Green y Edward Kaplan, "The Illusion of Learning from Observational Research", en Ian Shapiro, Rogers Smith y Tarek Masoud (eds.), Problems and Methods in the Study of Politics, Cambridge, Cambridge University Press, 2014.

Gibson, Edward, Boundary Control: Subnational Authorianism in Federal Democracies, Cambridge, Cambridge University Press, 2012.

Hall, Peter y David Soskice, Varieties of Capitalism, Oxford, Oxford University Press, 2001

Hall, Peter, "The Dilemmas of Contemporary Social Science", Boundary 2, vol. 34, núm. 3, otoño de 2007.

Humphreys, Macartan y Jeremy Weinstein, "Field Experiments and the Political Economy of Development", Annual Review of Political Science, vol. 12, 2009.

Kamola, Isaac y Neema Noori, "Higher Education and World Politics", PS: Political Science Ẽ Politics, vol. 47, núm. 3, julio de 2014, p. 599. 
Levitsky, Steven, Transforming Labor-Based Parties in Latin America, Cambridge University Press, 2003.

Levitsky, Steven y M. Victoria Murillo, "The Causes and Implications of Variation in Institutional Strength", Annual Review of Political Science, vol. 12, 2009.

, "Building Institutions on Weak Foundations: Lessons from Latin America", en Daniel Brinks, Marcelo Leiras y Scott Mainwaring (eds.), Reflections on Uneven Democracies: The Legacy of Guillermo O'Donnell, Baltimore, The Johns Hopkins University Press, 2014.

Levitt, Theodore, "Globalization of Markets", Harvard Business Review, mayo-junio de 1983.

Luna, Juan Pablo, María Victoria Murillo y Andrew Schrank, "Latin American Political Economy: Making Sense of a New Reality", Latin American Politics and Society, vol. 56, núm. 1.

Noori, Neema, "Does Academic Freedom Globalize? The Diffusion of the American Model of Education to the Middle East and Academic Freedom", PS: Political Science E Politics, vol. 47, núm. 3, julio de 2014.

O'Donnell, Guillermo, Modernization and Bureaucratic Authoritarianism: Studies on South American Politics, Berkeley, University of California, 1973.

O’Donnell, Guillermo y Phillip Schmitter, Transitions from Authoritarian Rule: Tentative Conclusions about Uncertain Democracies, Baltimore, The Johns Hopkins University Press, 1986.

Princhett, Lant y Justin Sandefur, "Context Matters for Size: Why External Validity Claims and Development Practice Don't Mix", Center for Global Development, Washington, D. C.

Samuel Huntington, "The Clash of Civilizations?”, Foreign Affairs, vol. 72, núm. 3, verano de 1993.

Shapiro, Smith y Masoud (eds.), Problems and Methods in the Study of Politics, Cambridge, Cambridge University Press, 2014.

Problems and Methods in the Study of Politics, Cambridge, Cambridge University Press, 2014.

Stallings, Barbara y Wilson Peres, Crecimiento, empleo y equidad: el impacto de las reformas economicas en America Latina y el Caribe, México, FCE / CEPAL, 2000. 
Svampa, Maristella, "Consenso de las 'commodities' y lenguajes de valoracion en America Latina”, Nueva Sociedad, núm. 244, marzo-abril de 2013, pp. 30-34.

Wallerstein, Immanuel, The Modern World System, Academic Press, 1974. 